calcique et chloruromercurique d'un même lait frais est constante et égale à 3,3 divisions du réfractomètre à immersion de Zisss $\left(N_{d}=0,0013\right)$

PANCHAUD $^{1}$ se livre à une étude comparative entre le sérum chloruromercurique d'AмвUHL et WeIss et le sérum chlorurocalcique d'ACKERMANN.

Le sérum chloruromercurique des laits acides est très clair et l'indice de ce sérum diminue avec le degré d'acidité du lait.

Cet auteur ne pense pas que le sérum chloruromercurique soit appelé à remplacer le sérum chlorurocalcique.

PaNCHAdD ${ }^{2}$ recherchant la cause de l'augmentation de l'indice de réfraction du sérum chlorurocalcique des laits aigris trouve qu' elle est due principalement à la formation de lactate de chaux aux dépens de la ehaux de la easéine. Le lactate de chaux reste en solution dans le sérum, en élève la réfraction jusqu'à ce que toute la chaux de la caséine soit saturée. Ce point atteint, la réfraction baisse, reprend la valeur qu'elle avait primitivement pour le lait frais, puis diminue encore ».

(A suivre).

\title{
RECHERCHES SUR LA VARIATION DE LA LACTATION DES VACHES GUERNESEY EN FONCTION DU TEMPS
}

\author{
par W. L. GAINES.
}

(Suite)

\section{Etude des courbes individuelles.}

Il a semblé logique à l'auteur de ce travail, au lieu de se borner à étudier la variation moyenne $d u$ rendement d'un groupe formé de nombreux animaux, d'entreprendre les déterminations des paramètres $A$ et $k$ relatifs aux équations exprimant les variations individuelles de rendement d'un certain nombre de sujets.

Pour les deux vaches dont les détails des rendements figurent sur le tableau $\mathrm{I}$, les valeurs numériques respectives de $A$ et de $k$ sont les suivantes :

$$
\begin{array}{lll}
\text { Vache No } 10233 & \mathrm{~A}=41.9 & \mathrm{k}=-0,002 \\
\text { Vache No } 10372 & \mathrm{~A}=74.5 & \mathrm{k}=-0,203
\end{array}
$$

Les écarts entre ces valeurs, et surtout celui qui sépare les valeurs de $\mathrm{k}$, sont importants. L'auteur a déterminé ces constantes pour un grand nombre d'animaux, afin de rechercher quelles étaient sous ce rapport les caractéristiques de la race de Guernesey.

La première étape de cette étude consista dans le choix de certains

1 L. Panchaud : Eitude sur les sérums chloromercurique et chlorocalcique du lait. Mitt. a. dem Geb. der Lebensmitteluntersuehung und Hygiene. XXII (1), 26,35, 1921.

2 L. PANCHAUD : Sur l'augmentation anormale de l'indice de réfraction du sérum chlorocalcique des laits aigris. Le Lait. V. 777-782, 1925. 
rendements, parmi les 15.000 résultats enregistrés au Herd-booki entre les années 1917 et 1922. Afin de ne pas compliquer la question, il ne fut tenu compte que des rendements enregistrés pendant 365 jours, sur des

\section{Figure 3}

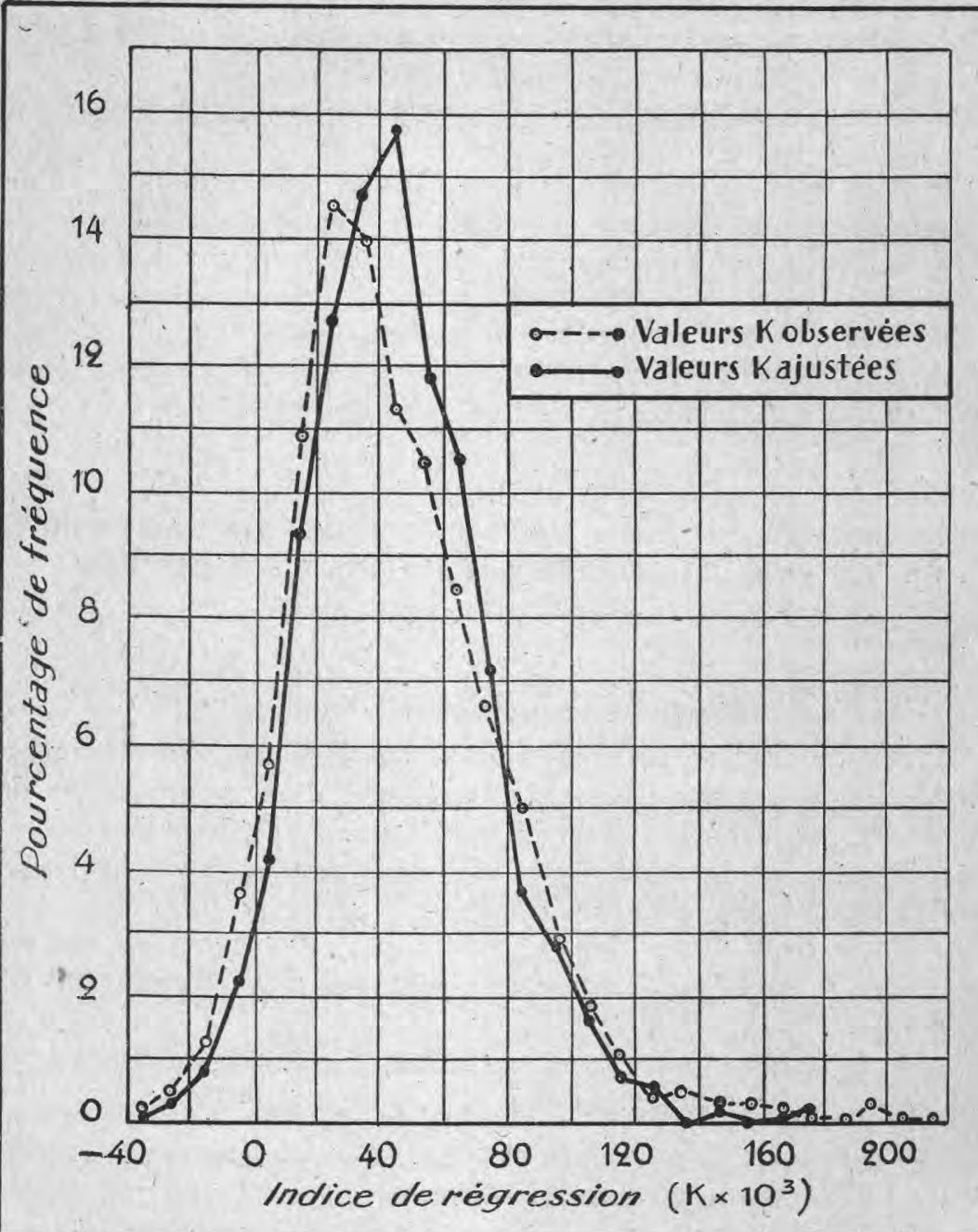

Courbe de fréquence des valeurs de l'indice de régression $k$.

vaches saillies après le sixième mois de lactation. Selon les données des Fig. 1 et 2, la gestation n'influence pas les rendements obtenus dans ces conditions. Le nombre des rendements sélectionnés de la sorte s'est élevé à 1534. 
La courbe de distribution des valeurs $A$ et $k$ calculée d'après ces données est très régulière et ne s'écarte qu'à peine d'une courbe de fréquence normale. La courbe des valeurs $k$ est représentée dans la Fig. 3, par le tracé discontinu et les cercles. Il importe de remarquer, qu'une proportion considérable $(5,4 \%)$ des rendements observés présentent des valeurs $k$, négatives, c'est-à-dire que le degré de sécrétion

Figure 4

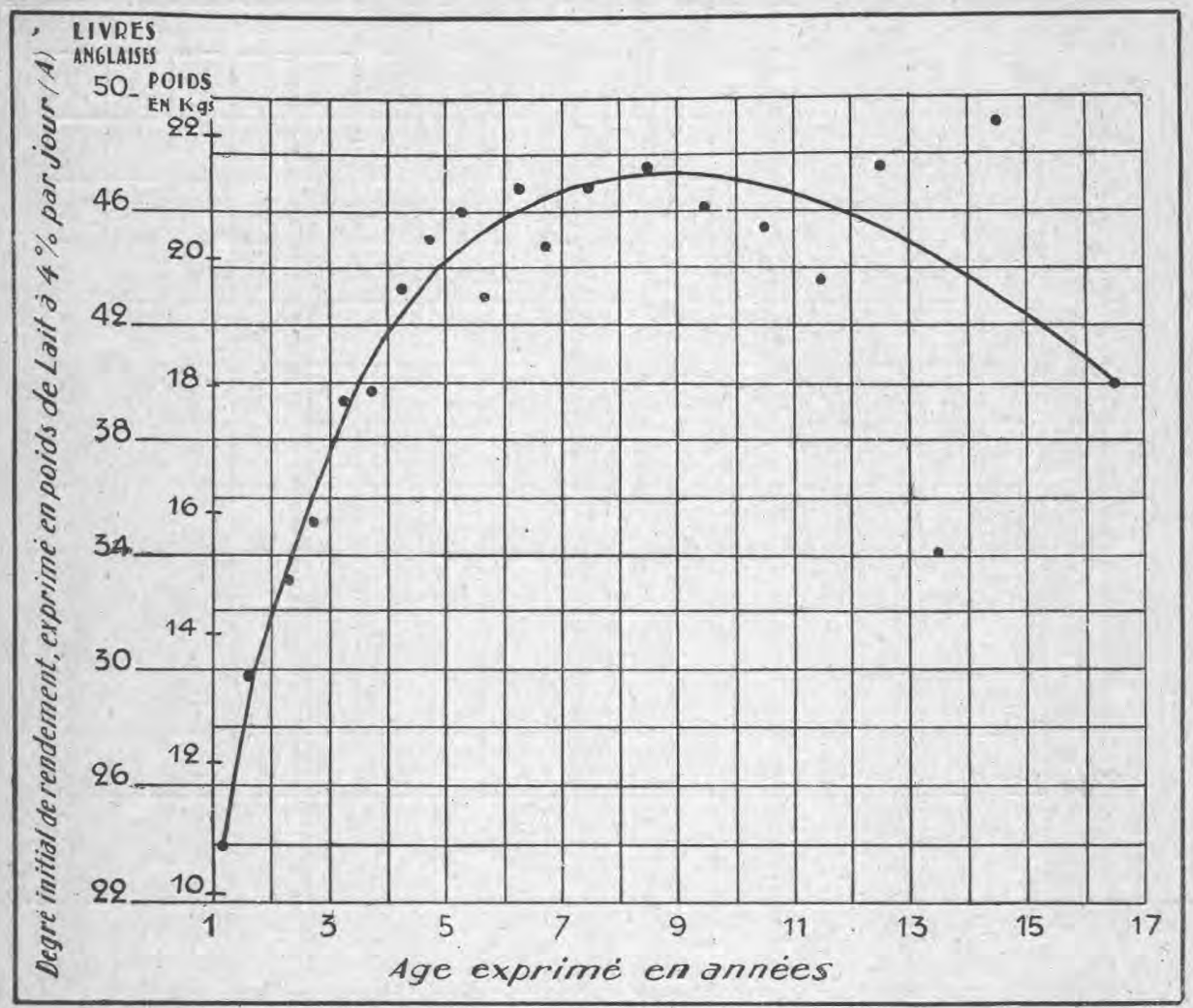

Variation du degré initial de rendement, $A$, en fonetion de l'âge de l'animal.

lactée augmente pour ces rendements avec la progression de la lactation, durant les onze premiers mois. La nature a donné à la vache la faculté de secréter du lait, destiné à subvenir à l'entretien du jeune bovin, durant la première période de son existence; mais il est certain, que l'homme a profondément modifié cette fonction, puisque l'activité de cette dernière peut se manifester dans toute son intensité durant une si longue période de temps.

Les données exposées plus loin montrent que les valeurs de $k$ sont nette- 
ment influencées par le degré initial de rendement A. De ce fait, on peut obtenir une conception plus adéquate de la earactéristique " regression ", lorsque les valeurs $k$ sont ajustées selon un degré initial de rendement type (Standard initial rate of yield). Ainsi ajustées, ces valeurs ont servi à établir la courbe de distribution exprimée par le tracé continu et les disques pleins de la Fig. 3. Les valeurs $k$ corrigées par cette méthode

Figure 5

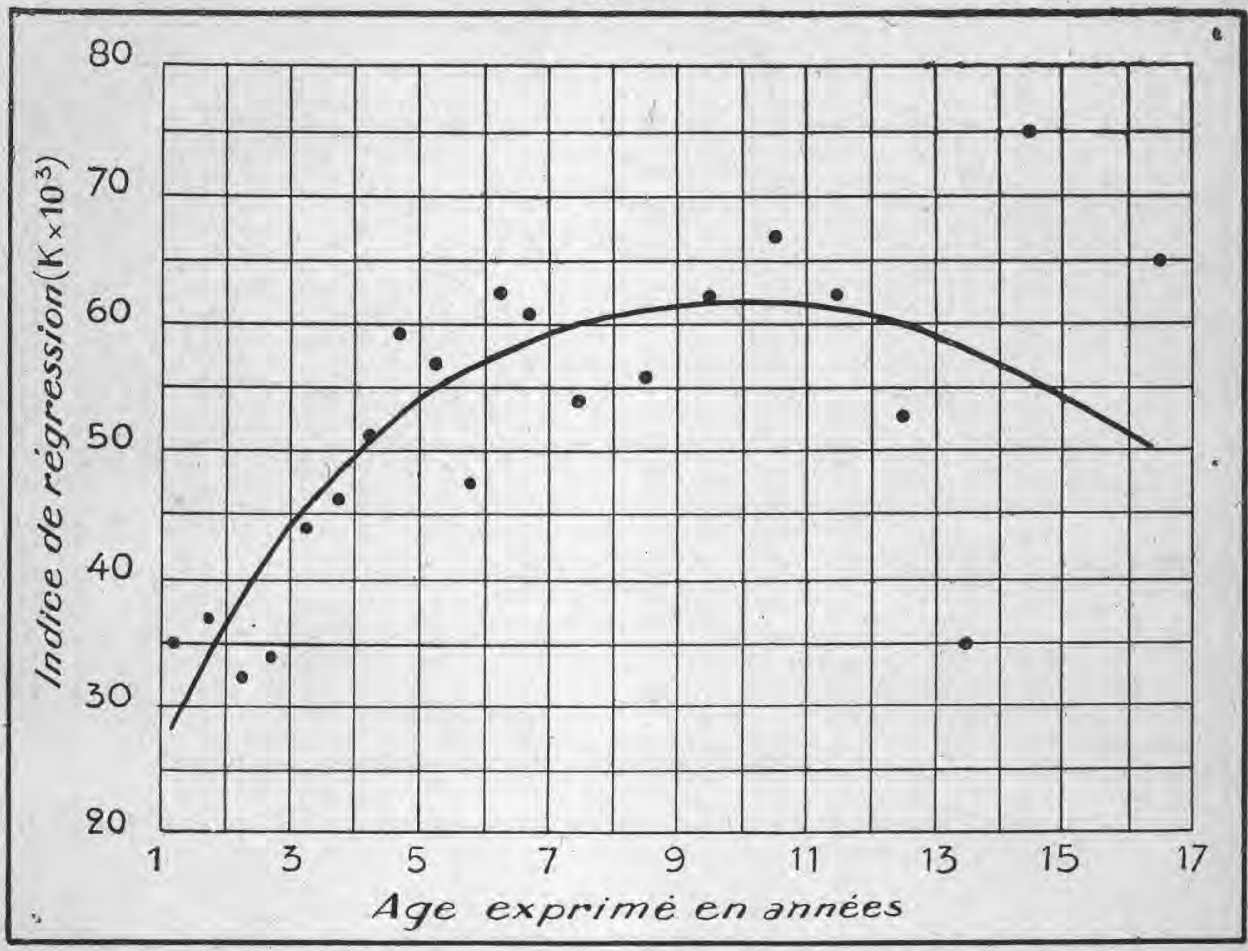

Variation de l'indice de régression, $k$, en fonction de l'âge de l'animal,

fournissent une courbe encore plus égale, plus régulière, que celle des valeurs de $k$ qui résultent de l'observation directe.

Il était intéressant d'étudier la variation en fonction de l'âge de l'animal de chacun des paramètres $A$ et $k$. Les résultats de cette étude sont résumés par les figures 4 et 5 .

La variation du degré initial de rendement, $\mathbf{A}$, peut-être interprétée comme un processus lié à celui du développement et de la senescence de l'organisme (Cf. Travaux de Brody et collabor. 11).

$\mathrm{Au}$ cours du développement corporel, il se produit un accroissement des dimensions et de la faculté de secrétion de la glande mammaire, lequel rend possible l'effort requis pour une lactation abondante. 
La réduction de $\mathrm{A}$, qui se produit un peu après la huitième année, est la manifestation de la senescence, appliquée à la mamelle.

Si nous considérons à ce point de vue la variation de l'indice de regression $k$, nous pouvons prévoir, à priori, qu'il faut une plus grande dépense d'énergie pour réaliser un faible degré de regression que pour obtenir une grande valeur absolue de ce facteur. Les valeurs élevées

\section{Figure 6}

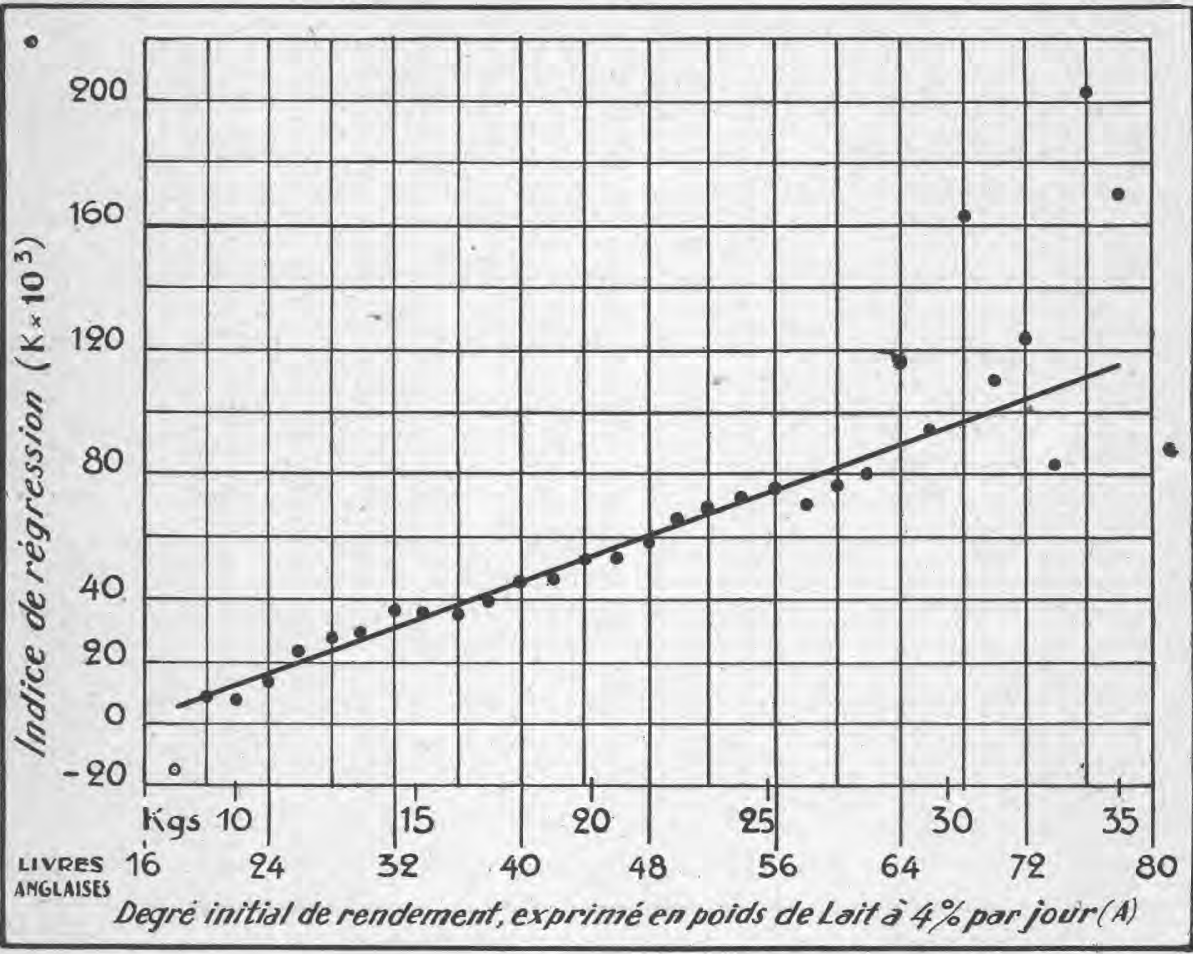

Relation entre le degré mitial de rendement, $A$, et l'jndice de régression, $k$. Sauf pour les rendements élevés, qui sont d'ailleurs les moins nombreux, cette relation peut s'exprimer par une équation linéaire, de la forme $k:=m A+n$.

de $k$ devraient correspondre, par conséquent, si cette hypothèse était exacte, aux vaches les plus faibles, c'est-à-dire les plus âgées. Or, on constate exactement l'inverse. Ici, il y aurait un processus de senescence précédant celui de développement, plutôt qu'un processus de développement suivi d'un processus de senescence.

L'auteur a calculé le cofficient de corrélation entre A, rendement initial, et $k$, indice de régression. Le résultat obtenu a été

$$
R_{\mathrm{sk}}=+0.535 \pm 0,012
$$

Il y a done une liaison nette entre les deux facteurs qui conditionnent 
le rendement en lait. La figure 6 exprime graphiquement cette liaison. La variation de $k$ en fonction de $A$ peut s'exprimer par une équation linéaire. Si nous classons ensemble les courbes des vaches qui présentent le même rendement initial, et si nous établissons pour chaque groupe la courbe qui représente la décroissance moyenne de rendement en fonction du temps, nous constatons qu'il existe une influence notable du degré initial de rendement A sur l'inclinaison de la courbe de rendement correspondante (voir à ce sujet le document 7).

Dix mois après le vêlage, des vaches, ayant un rendement initial de 80 livres de lait à $4 \%$ par jour, ont un rendement quotidien inférieur à celui de vaches, ayant un rendement initial de 40 livres par jour.

Quelle éventualité est préférable : un rendement abondant au début de la lactation et un défaut de persistance, ou un rendement moins abondant au début et une grande persistance ? Pour répondre à cette question, il n'y a qu'à calculer les coefficients de corrélation entre A, d'une part, $k$ d'autre part, et le rendement annuel total $Y$.

Le calcul donne :

$$
\mathrm{R}_{\mathrm{AY}}=0,672 \pm 0.009 \text { et } \mathrm{R}_{\mathrm{kX}}=0,226 \pm 0,016
$$

L'on voit ainsi que le rendement initial est ici plus important que le coefficient de régression.

Puisque le degré initial de rendement A exerce une action si importante sur l'indice de regression $k$, retournons à la figure 5 , et efforçons nous de voir comment varie le coefficient $k$ avec l'âge lorsque $\mathrm{A}$ reste constant.

Si nous considérons les observations faites sur des sujets de moins de 5 ans, qui représentent $72,6 \%$ du nombre total des résultats recueillis, nous pouvons déterminer les coefficients de corrélation ci-après :

$1^{\circ}$ Coefficient $R_{\mathrm{mA}}$, de l'âge $\mathrm{m}$ avec le rendement initial $\mathrm{A}$.

$2^{\circ}$ Coefficient $R_{\mathrm{mk}}$, de l'âge $\mathrm{m}$ avec l'indice de regression $k$.

$3^{\circ}$ Coefficient $R_{A k}$, du rendement initial A et de l'indice de regression qui sera nécessairement différent de celui que nous venons de caleuler précédemment, puisque le calcul est ici basé sur un moins grand nombre de données numériques.

On trouve dans ces différents cas :

$$
R_{\mathrm{mA}}=0,490 \pm 0,015 \quad \mathrm{R}_{\mathrm{mk}}=0,268 \pm 0,19 \quad \mathrm{R}_{\mathrm{Am}}=0,480 \pm 0,016
$$

De ces résultats, on peut tirer, par l'emploi de la méthode des corrélations partielles.

$$
\mathrm{R}_{\text {mkA }}=0,42 \pm 0,020
$$

Pour les âges inférieurs à cinq ans, nous devons conclure que la parsistanse est indépendante de l'âge, si on fait abstraction de l'influence de l'âge sur le degré initial de rendement. Les tracés réguliers des Fig. 4 et 5 suggèrent que cette caractéristique se maintient sans doute à tous les âges. 
L'examen de la Fig. 5 montre que les valeurs de $k$ peuvent servir de mesure, d'abord de la senescence, et en second lieu, du développement.

\section{Figure 7}

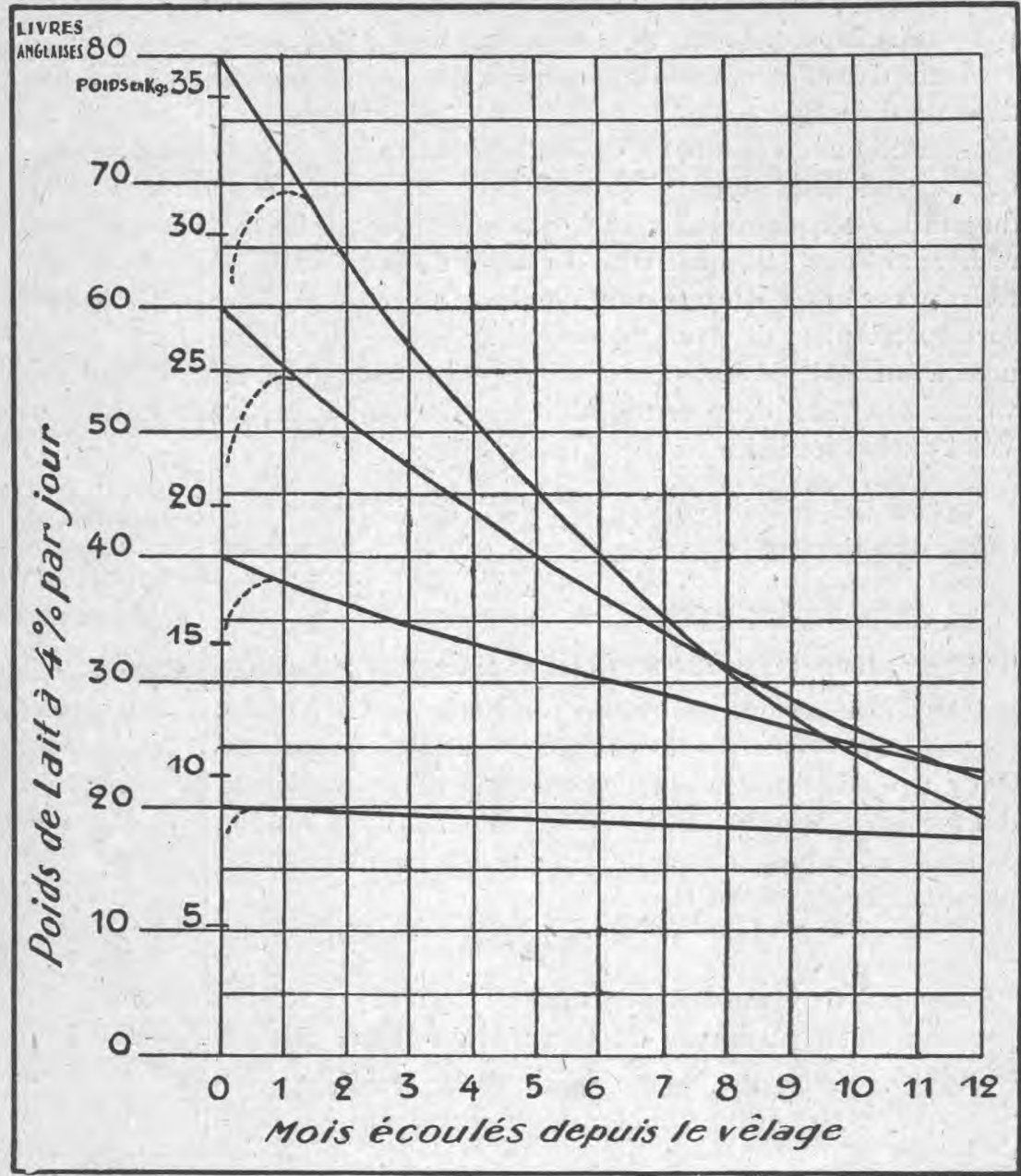

Ce document exprime, sous une autre forme, l'existence d'une relation entre $A$, rendement initial, et $k$, indice de régression. - Les courbes qui partent le plus haut ( $A$ élevé), se rapprochent plus rapidement que les autres de l'horizontale de base (valeurs correspondantes de $k$ plus élevées).

Si nous nous basons sur le coefficient de corrélation partielle, nous devons conclure que la valeur $k$ ne varie pas, si le degré initial de rendement demeure constant. Jusqu'à présent, rien ne peut expliquer ce fait puisque la présence d'uri faible indice de regression doit logiquement 
exiger une notable dépense d'énergie pour se manifester. On pouvait s'attendre à trouver une valeur de $k$ plus faible pour les vaches arrivées à l'âge du rendement maximum que pour les vaches plus jeunes, moins bien développées, mais l'on est bien obligé de constater qu'il n'en est pas ainsi. Apparemment, ni le développement, ni la senescence n'interviennent dans l'évolution du facteur $k$, bien que ce facteur soit lié à une dépense d'énergie.

Examinons maintenant, et d'une manière brève, si les caractéristi. ques, "rendement initial ), exprimés par $\mathrm{A}$, et "regression en fonction du temps », exprimée par $k$, sont transmissibles par hérédité. Nous avons recherché pour cela quel était le degré de corrélation entre les caractéristiques $\mathrm{A}$ et $k$ d'un certain nombre de vaches et les caractéristiques correspondantes de leurs demi-sœurs paternelles. Par comparaison, nous avons calculé les coefficients de corrélation entre $\mathrm{A}$ et $k$ pour des vaches sans lien de parenté, mais appartenant à un même troupeau. Voici quels furent les résultats enregistrés :

Vaches demi-sœurs

(issues d'un même père, et provenant d'un mềme troupeau)

Vaches non apparentées (provenant d'un même troupeau)
Correlation entre les valeurs de $A$

$$
\begin{array}{ll}
\mathrm{R}_{\mathrm{AA}}=0,267 \pm 0.027 & \mathrm{R}_{\mathrm{kk}}=0,157 \pm 0.029 \\
\mathrm{R}_{\mathrm{AA}}=0,100 \pm 0,019 & \mathrm{R}_{\mathrm{kk}}=0,158 \pm 0,019
\end{array}
$$

Ces données montrent que le " rendement initial " paraît transmissible, jusqu'à un certain point, par un mécanisme héréditaire. Par contre, il n'y a pas de différence, en ce qui concerne le coefficient de corrélation des facteurs $k$, entre les demi-soeurs et les sujets sans lien de parenté, ce qui tend à prouver que la "regression en fonction du temps " n'est pas un caractère héréditaire.

\section{- Résumé}

Le cours de la lactation de vaches Guernesey, à rendement enregistré par le système américain du Contrôle du Rendement, a été étudié en adaptant l'équation : $y_{m}=A e-k t$ au rendement énergétique mensuel de la mamelle, exprimé au moyen de quantités variables de lait à $4 \%$ de matière grasse. Dans cette équation, $y_{m}$ est le rendement d'énergie pour le mois dont le milieu est distant de $t$ mois du vêlage. Les constantes limitantes, A et $k$, ont une signification physiologique ; A représente la capacité de secréter du lait, au début de la lactation (rendement initial); $k$ représente le degré de régression de cette capacité, qui se produit au cours de la lactation (indice de régression en fonction du temps). L'équation a été d'abord utilisée pour examiner la moyenne de rendement de certains groupes de vaches; ensuite, pour examiner un certain nombre de cas individuels.

Les courbes de lactation des groupes indiquent que la gestation n'a 
pas d'influence appréciable sur le degré de secrétion lactée, durant les cinq premiers mois qui suivent la fécondation. Durant les stades ultérieurs, il y a une réduction appréciable, qui peut être attribuée à la croissance du foetus. L'étude des courbes de lactations individuelles montre qque les tracés qui expriment la fréquence de distribution des paramètres $\mathrm{A}$ et $\mathrm{k}$ ont une forme très régulière. La caractéristique $\mathrm{A}$ est un témoin des prozessus de développement et de senescence qui s'observent au cours de l'avance en âge ; la caractéristique $\mathrm{k}$, dont la manifestation exige une grande dépense d'énergie, paraît ne pas avoir de rapports avee le développement et la sénescence.

La corrélation entre le rendement d'une année et A est bien plus étroite, que celle entre ce rendement et $k$. La corrélation entre les caractéristiques $\mathrm{A}$ et $k$ est elle-même très étroite. Des vaches demisœurs, filles du même père, présentent, jusqu'à un certain point, une ressemblance entre elles, pour ce qui concerne la caractéristique A; mais, par contre, l'indice $\mathrm{k}$ ne semble pas transmissible par hérédité. Traduction R.N. GöRANSSON, revue par A.M. LERox.

\section{Bibliographie}

1. Gatnes, W.L. and Davidson, F.A. - Rate of Milk Secretion as Affected by Advance in Lactation and Gestation. III. Agr. Exp. Sta. Bul. 272, 1926.

2. Gaines, W.L. and Davidson, F.A. - The Effect of Advance in Lactation and Gestation on Mammary Activity. Journal General Physiology, Jan. 20, 1926, Vol, 9, pp. 325-332.

3. Gatnes W.L. - Persistency of Lactation in Dairy Cows. III. Agr. Exp. Sta. Bul. 288, 1927.

4. Garnes W.L. - Interpretation of the Lactation Curve. Journal General Physiology, Sept. 20, 1926, Vol, 10, pp. 27-31.

5. Garnes W.L. Measures of Persistency of Lactation. Journal of Agricultural Research, Feb. 15, 1927, Vol. 34, pp. 373-383.

6. Garnes W.L. - Milk Yield in Relation to Recurrence of Conception. Journal Dairy Science, March 1927, Vol. 10, pp. 117-125.

7. Brody S., Ragsdale A.C., and Turner W.C. - The Rate of Decline of Milk Secretion with the Advance of the Period of Lactation. Journal General Physiology, March 20, 1923, Vol, 5, pp. 441-444.

8. Gatnes W.L. and Davidoson F. A. - Relation Between Percentage Fat Content and Yield of Milk.III. Agr. Exp. Sta. Bul. 245, 1923.

9. Oterman O.R. and Sanmann F.P. - Valeur du Lait en Calories. Le Lait, Fev. 1927, Vol. 7, pp. 149-161.

10. Andersern A.C. - Undersogelser over Komaelkens Sammensaetning. Beretning fra N.J.F.s. Kongres i Oslo, Juni, 1926.

11. Brody S., Ragsdale A.C., and Turner C.W. - The Rate of Growth of the Dairy Cow. Journal General Physiology, Sept, 20, 1923, Vol. 6, pp. 31-40. 